\title{
Review of: "Graphene-based metasurface solar absorber design with absorption prediction using machine learning"
}

Tao Fu

Potential competing interests: The author(s) declared that no potential competing interests exist.

This MS proposed a graphene-based solar absorber to improve the absorption of the solar absorber. They used machine learning to predict the absorption properties of graphene metasurfaces. The work of this paper is clear and logical. However, there is no significant innovation from the content of the design, theory, and machine learning algorithm. It did not add a new and important result to the field.

1. In Figure 1, the differences between two figures are not labeled significantly, some import messages have been missed.

2. The first structure is a square ring rather than an O-shaped structure, so naming it as an O-shaped structure seems to be strong.

3. The symmetry and asymmetry of the structure are emphasized earlier in the article, but the effect of this property on the absorption rate is not analyzed later, and there is a lack of support from the references. I am puzzled by emphasizing the relationship between symmetry and asymmetry. Logically, the comparisons should be illustrated from beginning to end or an optimized structure shows the underlying characteristics.

4. The explanation of the relationship between the electric field diagram and the absorption rate is too simple and feeble. The underlying physics of the improvement has insufficient illustration. (Figure 3.)

5. Last but not the least, although authors used machine learning to predict the absorption result and the results show preferable value for optimizing the absorption. However, the mature tech of CNN does not demonstrate good innovation. Machine learning has a wide range of applications on graphene and metasurfaces, but it does not seem to make much sense to predict the absorption by these simple structural parameters. What's more, this MS does not end with a good theoretical analysis of the machine learning results. Mere statements of results without conclusions and theories are invalid. 